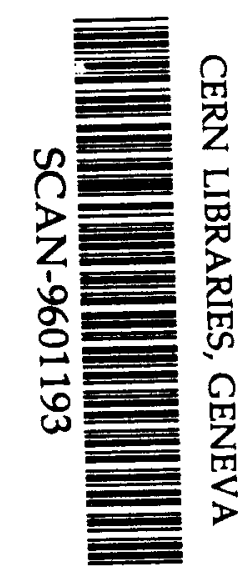

Sugg 605

\title{
Compressibility probed by linear momentum transfer
}

F. Haddad, J.B. Natowitz, B. Jouault, V. de la Mota, G. Royer and F. Sébille

Rapport Interne SUBATECH -95-16 


\title{
Compressibility probed by linear momentum transfer
}

F. Haddad ${ }^{1,2}$, J.B.Natowitz ${ }^{1}$, B.Jouault ${ }^{2}$, V. de la Mota ${ }^{2}$, G.Royer $^{2}$ and F. Sébille ${ }^{2}$

${ }^{1}$ Cyclotron Institute, Texas A $\&$ M University, College Station, Texas 77843, USA

${ }^{2}$ Subatech, 4 Av A. Kastler, 44070 Nantes cedex 03, France

(October 20, 1995)

\begin{abstract}
The linear momentum transfer occurring in central heavy ion collisions is studied within the Landau Vlasov model. A sensitivity to the Equation Of State is found for this observable. Assuming the free nucleon-nucleon cross section, a soft compressibility modulus (around $210 \mathrm{MeV}$ ) is required to reproduce the large amount of data available for linear momentum transfer. This result stands for local and non local effective potentials.
\end{abstract}

PACS numbers:25.70-z, 24.10Cn 
Heavy ion collisions offer the unique opportunity to explore nuclear matter under wide ranges of compression [1] and/or excitation energy [2]. It is then possible to drive the system far from its ground state and to study the thermodynamics of nuclear matter. Intensive work is being done both experimentally and theoretically to extract the Nuclear Equation Of State (EOS). Particular care is devoted to determine the compressibility modulus, $K_{\infty}$, which governs the ability of the nuclear matter to be compressed. This special interest is further motivated by the importance of this quantity in other fields of physics such as astrophysics [3].

The complexity of heavy ion collisions requires detailed theoretical analysis. Strong dynamical effects are present and a dual sensitivity to both, the nuclear equation of state and the in-medium nucleon-nucleon cross section, $\sigma_{n n}^{\text {med }}$, exists for many observables $[4,6]$. In addition, between 20 and several hundred $\mathrm{AMeV}$ they are of comparable importance which makes the extraction of the EOS difficult in this energy range.

In order to be sensitive to the EOS, an observable has to be developed at the beginning of the reaction when compression is present. It also has to be resistant to the evaporative stage of the reaction. The flow of nuclear matter [7] and the oscillation of the giant monopole resonance [8] are good examples. The linear momentum transfer in central collisions has also been suggested as a relevant observable and has been shown to be sensitive to both the equation of state and the nucleon-nucleon cross section [9]. This observable is established just after the preequilibrium stage of the reaction and contains information about early time of the collision. It is commonly used to characterize the transfer of the incident energy to the Compound Nucleus $(\mathrm{CN})$ formed in the reaction.

In this paper, the linear momentum transfer in central collisions is investigated within the Landau Vlasov model using different types of one-body effective potential.

Our calculations were performed by solving the Landau Vlasov equation [10] which describes the time evolution of the one-body phase space distribution function, $f(\vec{r}, \vec{p}, t)$ :

$$
\frac{\partial f(\vec{r}, \vec{p}, t)}{\partial t}+\{f(\vec{r}, \vec{p}, t), H\}=I_{\text {coll }}(f(\vec{r}, \vec{p}, t))
$$


where $\{$,$\} stands for the Poisson bracket, \mathrm{H}$ is the one-body mean field Hamiltonian and $I_{\text {coll }}$ is the Uehling-Uhlenbeck collision term.

Two main ingredients are needed in this transport model:

- an effective potential

- the in-medium nucleon-nucleon cross section.

As already stated in the introduction, these two features act together and their relative contributions to the evolution of the colliding system are important to know.

In spite of intensive theoretical work, in-medium effects on the nucleon-nucleon cross section remain an open problem [11]. We choose to work with the energy dependent free nucleon-nucleon cross section in all our calculations. This choice is based on early work on the flow observable [4-6]. Indeed, Bao-an $\mathrm{Li}$ found [4] that measurements of the balance energy, where the flow vanishes, are accurately reproduced using a local Zamick interaction and the free nucleon-nucleon cross section. Also, a study of flow in $\mathrm{Nb}+\mathrm{Nb}$ reactions from 50 to several hundred $\mathrm{AMeV}$ showed a good agreement of the calculation with the data when a momentum dependent interaction was used together with the free nucleon-nucleon cross section [6].

In order to study EOS effects, we used different types of potential.

- Local Zamick interactions with an isospin dependence and $K_{\infty}=200 \mathrm{MeV}$ (soft) and $K_{\infty}=380 \mathrm{MeV}$ (stiff) respectively.

- Momentum dependent interactions based on the Gogny interaction [12] with soft $\left(\mathrm{D} 1 \mathrm{G} 1, K_{\infty}=228 \mathrm{MeV}\right)$ and stiff $\left(\mathrm{D} 1 \mathrm{G} 3, K_{\infty}=360 \mathrm{MeV}\right)$ compressibility.

Functional forms and coefficients of these potentials can be found in ref [6].

In order to determine the linear momentum of the $\mathrm{CN}$ in central collisions, the first step is to look at the emission pattern of the reaction. Such a typical pattern is displayed in the upper panel of Figure 1 for the $\mathrm{O}+\mathrm{U}$ reaction at $50 \mathrm{AMeV}$ and $\mathrm{b}=2 \mathrm{fm}$. It corresponds to 
a LV calculation using a local soft interaction. It should be noted that similar results are obtained for any of the other effective forces used in this work.

The interpenetration stage begins around $t_{0}=30 \mathrm{fm} / \mathrm{c}$ and from there the thermalization occurs. This is expressed in the lower panel of Figure 1 which presents as a function of time the normalized quadrupole moment in p-space of the full distribution. The normalized quadrupole moment reaches zero around $t_{1}=140 \mathrm{fm} / \mathrm{c}$. This means a spherical distribution in p-space, ie, global thermalization is achieved. Between $t_{0}$ and $t_{1}$ preequilibrium emission occurs. In our calculations, free particles are identified as those nucleons experiencing a local density lower than $\rho_{0} / 10$. In this work, the $\mathrm{CN}$ is assumed to be formed once the preequilibrium stage is finished. All CN characteristics are then determined at $t_{1}$.

We choose to focus on central collision (impact parameter between 0 and $4 \mathrm{fm}$ ) of the ${ }^{16} \mathrm{O}+{ }^{238} \mathrm{U}$ system at several energies (ranging from 20 to $70 \mathrm{AMeV}$ ). In Figure 2, the linear momentum transfer calculated for the Zamick interaction is displayed as a function of the quantity $\sqrt{\left(E-V_{c}\right) / A_{p}}$ where $V_{c}$ represents the Coulomb barrier between the target and the projectile, $\mathrm{E}$ is the incident energy and $A_{p}$ is the projectile mass number. Symbols correspond to experimental data [14-25]. Theoretical results for central collisions are represented by thick bars. The length of these bars accounts for the different impact parameter values selected as central collisions. Results using the Soft EOS are represented by black bars whereas those from the stiff EOS are represented by shades bars.

The calculations with Zamick soft interaction follow nicely the experimental values over a wide range of incident energies. On the other hand, the calculations with the Zamick stiff interaction overestimate the momentum transfer. This finding is in accordance with that of ref [9] and can be compared with a previous work of Xu et al [13] which found that a local stiff interaction always overestimates the $\mathrm{CN}$ formation cross section.

In order to get deeper insight into this phenomenon, the momentum transfer per nucleon of the projectile as a function of the incident energy is reported in Figure 3. Solid symbols (points and triangles) represent our LV calculations. Data for ${ }^{14} N+{ }^{238} U$ are from ref [15] and are displayed as open circles. The experimental points show a saturation of the transferred 
momentum per nucleon around $200 \mathrm{MeV} / \mathrm{c}$ for incident energy above $30 \mathrm{AMeV}$. To clearly see the decrease of momentum transfer with incident energy, a line assuming full momentum transfer is also displayed on Figure 3. All this features are well reproduced by the Zamick soft interaction. For the Zamick stiff, a saturation is also present but the momentum transfer is overestimated by $25 \%$ (see triangles).

These results suggest that, assuming $\sigma_{n n}^{\text {med }}=\sigma_{n n}^{\text {fret }}$, a soft interaction is needed in order to reproduce basic dynamical nuclear properties such as linear momentum transfer.

Before any definite statement on the value of the compressibility modulus is made, the effect of the momentum dependence of the mean potential has to be studied. This dependence is known to have important effects on the flow observable in this energy range. Indeed the inclusion of such dependence drastically changes the conclusions on the compressibility modulus value. Use of local interactions imply a stiff EOS in [26] whereas inclusion of momentum interaction favor softer ones $[7,6]$.

Figure 4 summarizes calculations obtained for Gogny type interactions. Results using the D1G1 force $\left(K_{\infty}=228 M \epsilon V\right)$ are displayed by thick black bars and those from D1G3 $\left(K_{\infty}=\right.$ $360 \mathrm{MeV}$ ) by thick shaded bars. For momentum dependent interaction, the difference between the results using the two different compressibility values is smaller. D1G3 overshoots the data quite clearly. D1G1 on other hand is in agreement with the data although it slightly overestimates them at high energy. Nevertheless, the soft EOS is again favoured for momentum dependent interaction.

In conclusion, calculations within the Landau Vlasov framework have been performed to study the linear momentum transfer in heavy ion reaction. In accordance with ref [9] a sensitivity of this observable to the EOS has been observed. A detailed study confirms the need of a soft EOS in order to reproduce the large amount of data available for linear momentum transfer. This conclusion is valid for local as well as for momentum dependent interactions. This result is in agreement with several previous calculations $[7,8,6]$ which find an compressibility modulus value around $210 \mathrm{MeV}$. In medium effects which are believed not to change by more than $20 \%$ the free cross section [11] should only slightly modify our 
results.

\section{ACKNOWLEDGMENTS}

This work was supported by the U. S. Department of Energy under Grant DE-FG0586ER40256, the National Science Foundation, The Robert A. Welch Foundation and the french Centre National de la Recherche Scientifique. 


\section{REFERENCES}

[1] B.Kampfer et al, Phys. Rev. C48, R955(1993).

[2] V.Metivier et al, preprint LPCC 95-06.

[3] J.Cooperstein and E.A.Baron in: Supernovae, ed A.Petschek (Springer, Berlin,1990) 213.

[4] Bao-an Li, Phys. Rev. C48, 2415(1993).

[5] V. de la Mota et al, Phys Rev C46, 677(1992).

[6] F.Haddad,F.Sébille,M.Farine,P.Schuck,V.de la Mota and B.Jouault, Phys Rev C(in press).

[7] Q.Pan and P.Danielewicz, Phys. Rev Lett. 70, 2062(1993); Phys. Rev. Lett. 70, $3523(1993)$.

[8] J.P.Blaizot, Phys. Rep. 64, 173(1980).

[9] J.Cibor, J.Lukasik and Z. Majka, Z. Phys. A348, 233(1994).

[10] B.Remaud, C.Grégoire, F.Sébille and P.Schuck, Nucl. Phys. A488, 423c(1988).

[11] J.Cugnon, A.Lejeune and P.Grangé, Phys. Rev. C35, 861(1987).

[12] J.Dechargé and D.Gogny, Phys. Rev. C21, 1568(1980).

[13] H.M. Xu, W.G. Lynch, P.Danielewicz and G.F.Bertsch, Phys. Rev. Lett. 65, 843(1990).

[14] M.B. Tsang, D.R.Klesch, C.B.Chitwood, D.J.Fields, C.K.Gelbke, W.G. Lynch, H.Utsunomiya, K.Kwiatkowski, V.E.Viola and M.Fatyga, Phys. Lett. B134, 169(1984).

[15] M.Fatyga, K.Kwiatkowski, V.E.Viola, C.B.Chitwood, D.J.Fields, C.K.Gelbke, W.G. Lynch, J.Pochodzalla M.B. Tsang and M.Blann, Phys. Rev. Lett 55, 1376(1985).

[16] E.C.Pollaco, M.Conjeaud, S.Harar, C.Volant, Y.Cassagnou, R.Dayras, R.Legrain, 
M.S.Nguyen, H.Oeschler and F.Saint Laurent, Phys. Lett. B146, 29(1984).

[17] J.Galin et al, Phys. Rev. Lett. 48, 1787(1982).

[18] V.E.Viola, B.B.Back, K.L.Wolf. T.C.Awes, C.K.Gelbke and H.Breue, Phys. Rev. C26, 26(1982).

[19] A. Fahlhi, J.P.Coffin, G.Guillaume, B.Heush, F.Jundt, F.Rami, P.Wagner, P.Fintz, A.J.Cole, S.Kox and Y.Schutz. Phys. Rev. C34, 161(1986).

[20] J.Galin, G.Ingold, U.Jahnke, D.Hilscher, M.Lehmann, H.Rossner, and E.Schwinn, Z. Phys. A331, 63(1988).

[21] E.Duek, L.Kowalski, M.Rajagopalan, J.Alexander, D.Logan, M.S.Zisman and Morton Kaplan, Z. Phys A307, 221(1982).

[22] G.La Rana et al, Nucl. Phys A407, 233(1983).

[23] D.Utley, R.Wada, K.Hagel, J.Li, X.Bin, M.Gui, Y.Lou, R.Tezkratt, J.B.Natowitz and M.Gonin, Phys. Rev. C49, 1732(1994).

[24] K.Hagel et al, Nucl. Phys. A486, 429(1988).

[25] F.Saint Laurent, M.Conjeaud. R.Dayras, S.Harar, H.Oeschler and C.Volant, Phys. lett. B110, 372(1982).

[26] J.J.Molitoris and H.Stocker, Phys. Rev.C32, 346(1985). 


\section{FIGURES}

Figure 1

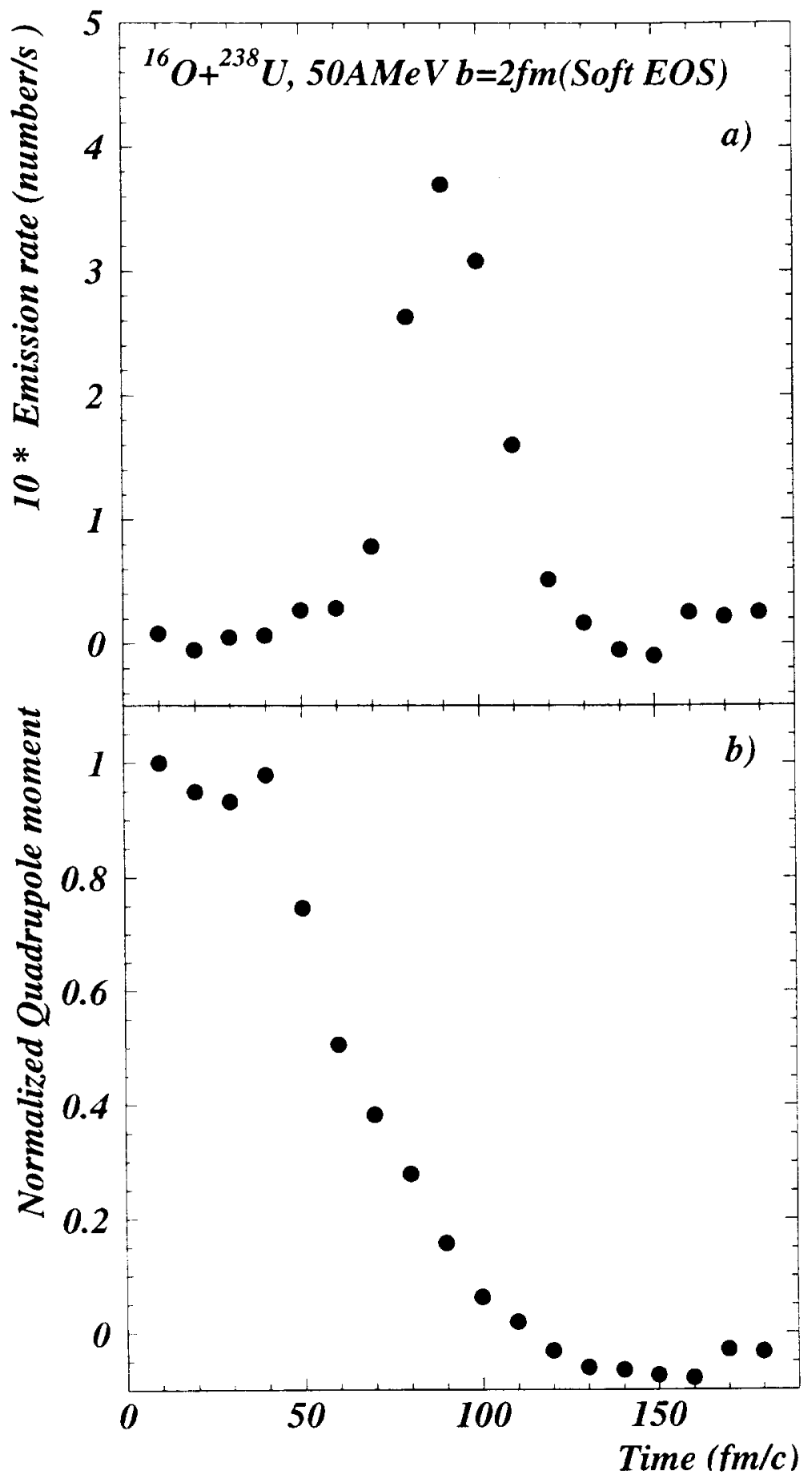

FIG. 1. Emission rate(upper panel) and normalized quadrupole moment (lower panel) as a function of time for ${ }^{16} \mathrm{O}+{ }^{238} \mathrm{U}$ at $50 \mathrm{AMeV}$ 
Figure 2

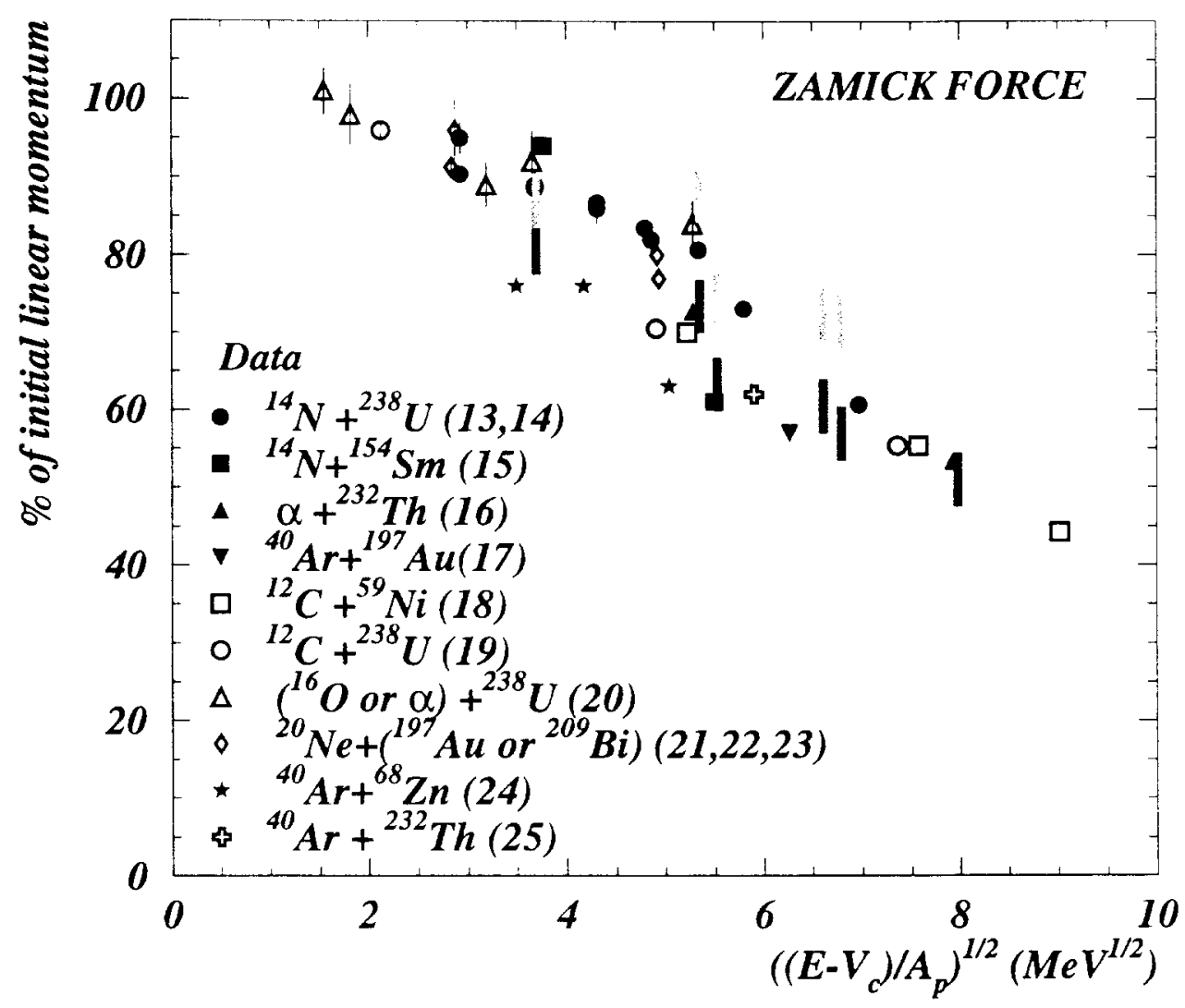

FIG. 2. Linear momentum transfer for local interactions. Calculations are represented by thick bars and data by symbols. 
Figure 3

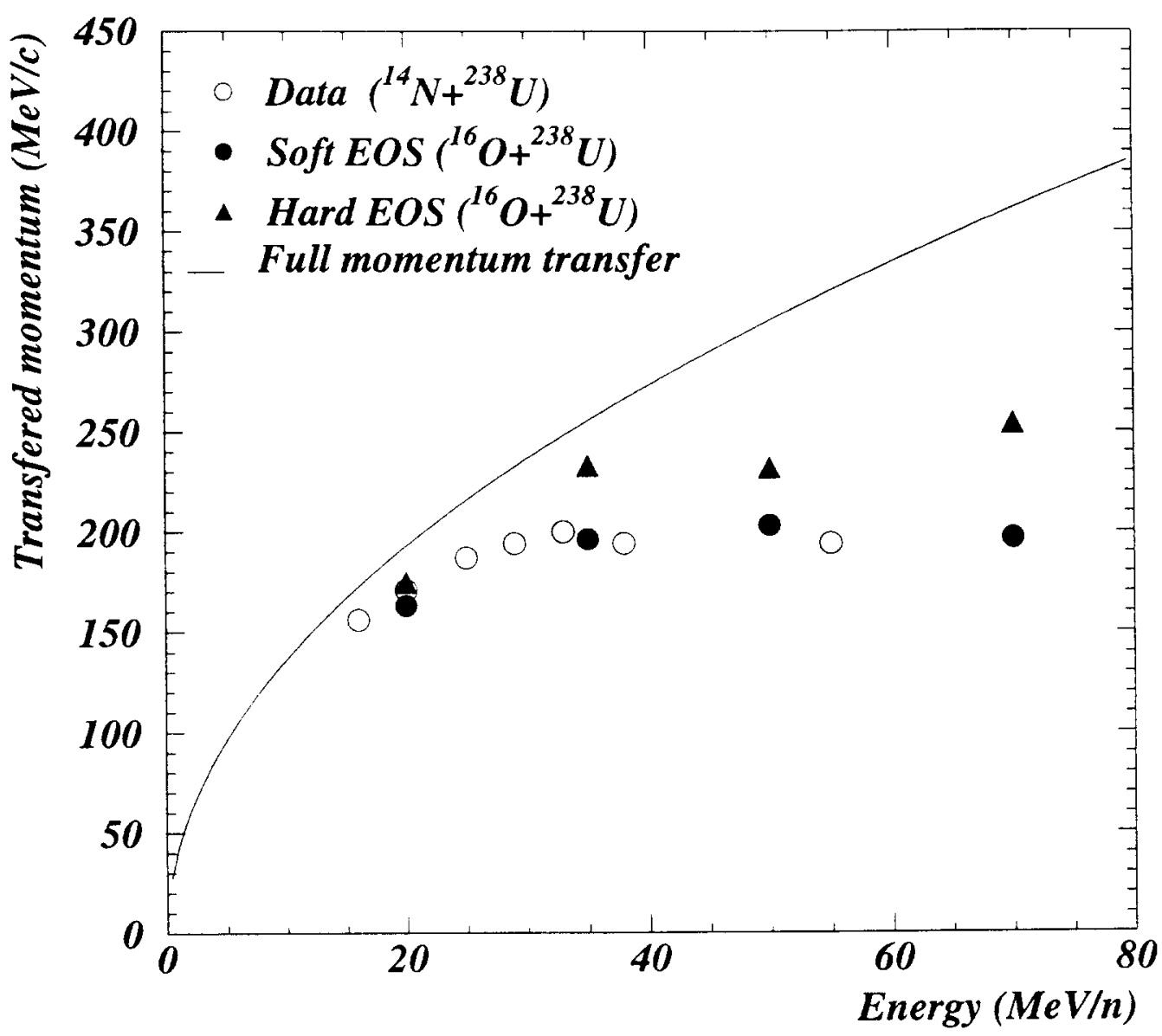

FIG. 3. Linear momentum transfer per incident nucleon as function of the energy for local interactions. Calculations correspond to solid symbols (points and triangles) and data to open circles. The curve characterizes full momentum transfer. 
Figure 4

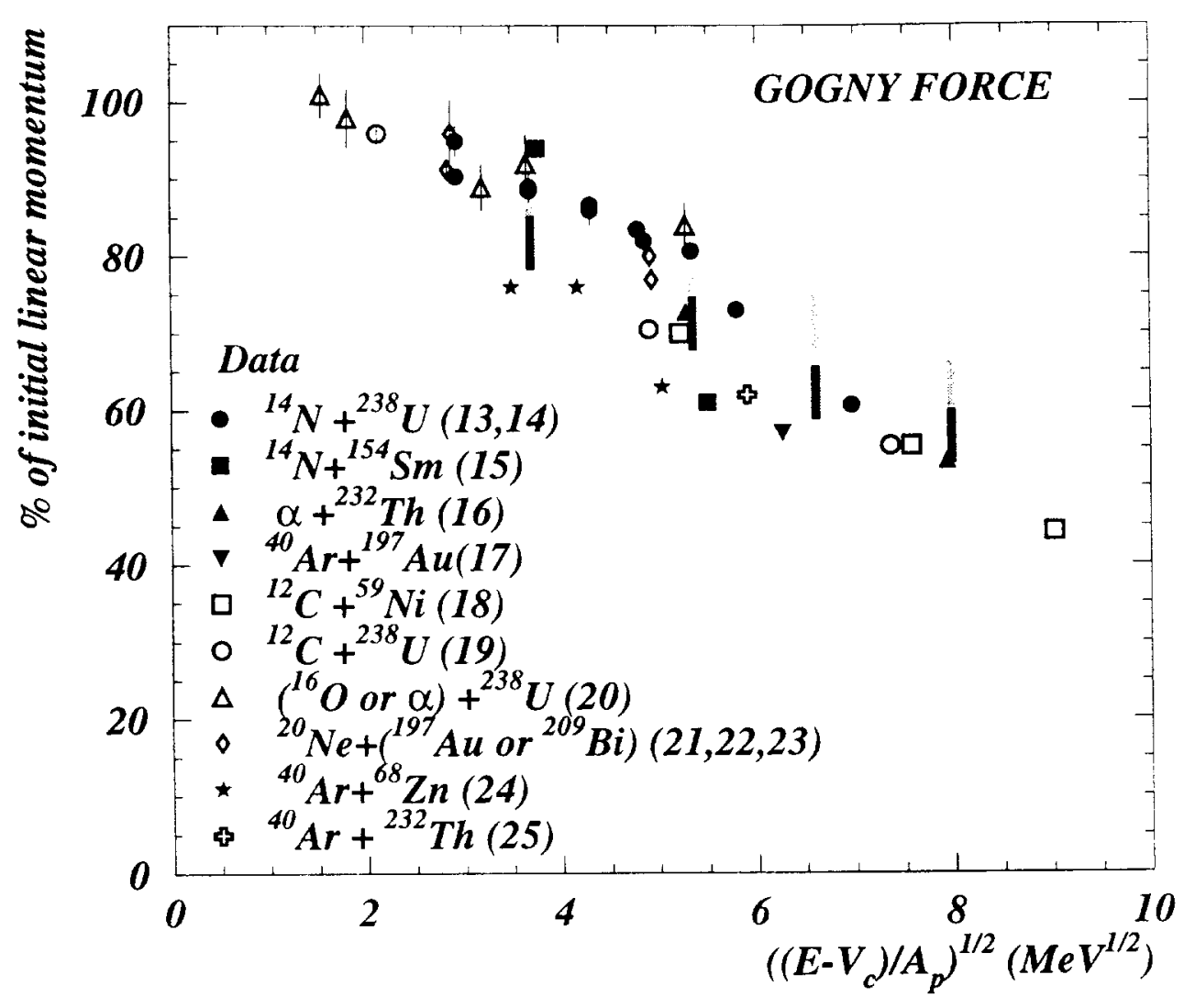

FIG. 4. Linear momentum transfer for momentum dependent interactions. Calculations are represented by thick bars and data by symbols. 